\title{
Comparison of the Effect of Exercise Type on the Prognosis Scores in Patients with Acute Ischemic Stroke
}

\author{
Mahshid Kadkhodaei Khalafi' ${ }^{1}$, Leila Simani ${ }^{2}$ (D), Maziar Shojaei ${ }^{*(1 D)}$, \\ Mohammadreza Hajiesmaeili ${ }^{4}$ iD
}

1. Dept.of Exercise Physiology,Loghman Hakim Hospital,Shahid Beheshti University of Medical Sciences, Tehran, Iran.

2. Skull Base Research Center, Loghman Hakim Hospital, Shahid Beheshti University of Medical Sciences, Tehran, Iran.

3. Dept. of Neurology, Loghman Hakim Hospital, Shahid Beheshti University of Medical Sciences, Tehran, Iran.

4. Anesthesiology Research Center, Loghman Hakim Hospital, Shahid Beheshti University of Medical Sciences, Tehran, Iran

\begin{tabular}{|c|}
\hline Article Info \\
\hline doi $10.30699 /$ jambs.29.135.215 \\
\hline $\begin{array}{l}\text { Received: 2020/06/10; } \\
\text { Accepted: 2020/12/19; } \\
\text { Published Online: } 28 \text { Feb 2021; }\end{array}$ \\
\hline $\begin{array}{l}\text { Use your device to scan and read the } \\
\text { article online }\end{array}$ \\
\hline 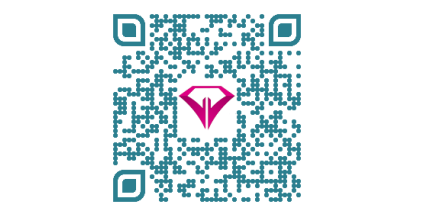 \\
\hline
\end{tabular}

Corresponding Information: Maziar Shojaei,

Dept. of Neurology, Loghman Hakim Hospital, Shahid Beheshti University of Medical Sciences, Tehran, Iran

E-Mail: : maziar.shojaei@gmail.com

\section{ABSTRACT}

Background \& Objective: Cerebrovascular accidents (CVA) are of the main causes of mortality and morbidity in the world. This study aimed to investigate the two training protocols in patients with acute ischemic stroke (AIS), and their relationship with the patients' prognosis.

Materials \& Methods: This experimental study included 45 patients whose ages ranged from 45 to 65 years. Patients were recruited by convenience sampling and purposive method. The participants were categorized into two intervention groups high-intensity anaerobic training (HIT) and continuous aerobic exercise). The control group individuals had no history of exercise. All three scores were assessed at the beginning of the study and after 28 days.

SPSS 22 was used to analyze the collected data and the following statistical tests were performed: independent samples t-test, ANOVA and Tukey post hoc test. The $\alpha$ level was considered to be 0.05 .

Results: The National Institutes of Health Stroke Scale (NIHSS) score reduced in both exercise groups compared to the controls, in the post-intervention period (frame 9 to 7). The Mini- Mental State Examination (MMSE) score also increased in the continuous exercise group (from 21.93 to 23.4 in the HIT group and from 22.4 to 24.14 in the continuous group), while the Modified Rankin Scale (MRS) score reduced (frame 3 to 2$)(P=0.001)$.

Conclusion: Performing intermittent exercise over four weeks can improve the overall prognosis quality of CVA patients by reducing the MRS score.

Keywords: Acute ischemic stroke, Neurological scores, Outcome measure, Type of exercise

\section{Introduction}

One of the major causes of lengthy disability is cerebrovascular accident (CVA) [1,2]. The suffered patients show various degrees of cognitive, learning and memory disorders and functional impairments in different fields, especially daily activities such as eating, drinking, writing, etc. $[3,4,5,6]$.

Exercise keeps the brain and nerve cells healthy by two ways: First, increasing the traction stress on the cerebral arteries wall and activating related signals and second, increasing the metabolic activity of neurons in the brain, which causes a series of intracellular events. The interaction of these two simultaneous mechanisms leads to an increase in endothelial and neuronal growth factors; it also increases antioxidant activity and intracellular cascade factors in mitochondria. The cited interaction changes the levels of brain-derived neurotrophic factors; as a result, cerebral blood flow increases.

The angiogenesis process can be examined by increasing the complexity of cerebral vessels and neuronal volume changes, which increase dendritic density, neuronal variability and vascular wall compliance. The cited events can improve a person's learning, memory and performance [7].

Various studies have been performed on the effects of exercise with different protocols on the Amyotrophic Lateral Syndrome (ALS) patients (e.g. Bllinger, 2014; Horn By; Sundseth, 2012; Vanroy, 2017; Pierce, 2018; Tang, 2010; Matsuda) $[1,2,8,9,10,11,12]$. A study by Pierce et al. (2016) showed that high-intensity and 
enduring aerobic exercises by a chronic ischemic stroke participant for four weeks, changed the oxygen intake and increased walking test time [13]. Similarly, a six-week advanced individual training (AIT) intervention for acute ischemic stroke (AIS) patients showed that high-intensity exercise was possible with safety maintenance. The participants experienced significant clinical progress in walking [14]. The progression is due to the endurance and high-intensity exercises (by bicycle), which change the performance, cognition and motivation of people to a better prognosis [15].

Therefore, high intensity training (HIT) may recover functionality in stroke rehabilitation. This training strategy can help or substitute the outdated moderateintensity exercise to support more active and prompt physiological versions. They disclosed that HIT may successfully recover the cardiovasculiatory fitness of stroke patients faster [16]. This study aimed to investigate the two training protocols in patients with AIS and below 6-24 hours, and their relationship with the patients' prognosis and behavioral and cognitive functions.

\section{Materials and Methods}

This clinical trial study was conducted on 93 patients diagnosed with an ischemic stroke. It concluded one control and two intervention groups. The patients were selected from the cases who referred to the emergency ward of Loghman Hakim Hospital in Tehran, from December to March 2018. patients with one of the following characteristics were excluded from the study: a history of drug abuse, coagulation disorders, cancer, advanced renal, hepatic, cardiac or respiratory failure, fever above $38^{\circ} \mathrm{C}$, musculoskeletal abnormalities, vascular aneurysm, history of ischemic stroke, open wound injury and concussion, an altered level of consciousness (ALOC) and mental retardation (based on their medical records).

After meeting the inclusion criteria and less than 24 hours after admission, 63 patients with a definitive diagnosis of ischemic stroke were selected, according to the para clinical tests (CT-scan) by a neurologist, the willingness to participate in the exercise program and Glasgow Coma Scale (GCS).

GCS contains three parts to estimate coma severity and depth of loss of consciousness in brain damaged patients over the age of five. It scores patients based on: eye opening $=4$, verbal response $=5$ and motor response $=6$ criteria. The maximum score is 15 (only applicable when all three components are testable), and the minimum is 3 (when all of component are not testable). Patients with the GCS scores of 12-15 (based on consciousness level) were selected to enter the study.

Among evaluated individuals, 45 patients aged 45-65 years were selected. The informed consent was signed and the general information and sports questionnaire forms were completed. Estimation the degree of disability following stroke was done based on the Modified Rankin Scale (MRS). The convenient and non-random sampling was performed. The participants were categorized into two intervention groups (15 patients in each group) and the control group (15 patients). All three groups were matched for ranges of their initial National Institutes of Health Stroke Scale (NIHSS) and MRS and stroke subtypes.

After familiarizing with the exercise environment and using rehabilitation bikes, the first experimental group, performed the aerobic periodic exercise (HIT) for three seconds with 4-6 repetitions and maximal oxygen consumption intensity of $70-80 \%\left(\mathrm{VO}_{2}\right.$ peak) (measured by chronometer and finger pulse oximetry devise) (formula-1) [31]. In the next step, they rested four and half minutes and cooled down two minutes with stretching exercises. This aerobic exercise was repeated three times a week. The workload was 225 kilo-joules based on the exercise protocol offered by Burgomaster in 2008 [17], in addition to the specific physiotherapy protocol.

After getting acclaimed with the exercise setting and warming-up (for two minutes), the second training group did an uninterrupted aerobic exercise by a rehabilitation bike with a severity of $60 \%-65 \%$ of the maximum submaximal consumed oxygen for 40-60 minutes a day, five days a week. Besides, the workload was $2250 \mathrm{KJ}$ based on the Martin Gibala (2020) exercise protocol [18]. After the ride, the participants did the cooling down for two minutes. The exercise lasted for 28 days (with a specific physiotherapy protocol).

The control group with 15 members (the physiotherapy protocol only) just used isometric training with Triband bond, Swiss Ball and hat pack for forty-five minutes in the morning (the standard medical without the use of tens and radiation were performed by one person). The workload was considered the same in both types of exercises. Also lactate was not measured in this study.

Formula-1: $\mathrm{VO}_{2}$ peak (volume of oxygen consumption peak oxygen uptake): $0.021(\text { time })^{3}-0.451(\text { time })^{2}+$ 1.379 (time) - 14.8 (ml/kg.min)

Foot and hand rehabilitation bikes were available in both large and fixed forms (German Turbo Fitness), for the patients to sit or lean, in the small and portable sizes (KeepFit, China). The bikes had handles to support the patient, equipped with a TV display to adjust the speed, duration and intensity and a remote control for the exerciser [19].

Besides, all patients continued their drug treatment for stroke following the doctor's instructions. They had the same diet and environmental conditions. All the subjects were checked by a heart monitoring equipped with an oximetry pulse to serially control the blood pressure, pulse and oxygen saturation percentage in the blood.

All procedures were performed in the occupational therapy clinic of Loghman Hakim Hospital Medical Center. To reduce errors and closely monitor the procedures, all training sessions were held with the presence of a doctor and the researcher. They were held in the same place, at 3-8 PM. The place temperature was 
$25^{\circ} \mathrm{C}$, with air humidity of $35-42$ degrees and air pressure of 700-1060 Pa (according to the reports by Tehran Province Meteorological Administration).

The MRS was used to assess patients' motor skills. This is a six-point disability scale with possible scores ranging from 0 (no residual symptoms) to 5 (severe disability). A separate category of six is usually added for patients, who pass away [20]. The NIHSS used to assess the participants' motor and speech performance. It is composed of 11 items, that score the range of $0-42(0=\mathrm{No}$ stroke symptoms, 1 to $4=$ Minor stroke, 5 to $15=$ Moderate stroke, 16 to $20=$ Moderate to severe stroke, and 21$42=$ Severe stroke).

No change in motor skills or low motor skills was considered as poor prognosis. Motor skills improvement was regarded as a good prognosis, therefore aphasic patients were excluded [21]. We used the 30-items Mini-Mental State Examination (MMSE) questionnaire to assess cognitive impairment as the research outcome, at the beginning and the end of study. All three scores were assessed at the beginning of the study and the end of 28 days.

Chi-square and Fisher's exact tests were used to evaluate the participants' qualitative and descriptive demographic data. Besides, the independent samples ttest, ANOVA, Tukey post hoc test were used at the $\alpha$ level of 0.05 , to analyze the values of each of the dependent variables in the pre- and post-exercise stages and between the two groups. The tables were also drawn using SPSS 22 (SPSS Inc., Chicago, IL., USA).

The study was approved by the ethics committee of Shahid Beheshti University of Medical Sciences and the following ethic code: IR.SBMU.RETECH.REC .1397.1018 and ID code IRCT 201005180039433N1.

\section{Results}

According to table 1, out of 63 participants, 18 patients (20\%) were excluded. Exclusion criteria are as follows: four patients died, two had a new stroke, nine left the study due to personal reasons, distance and impossibility to refer to the center and three were demotivated and not willing to take part in the study. The final sample consisted of 45 participants including 33 men (75.6\%) and 22 women $(24.4 \%)$, who completed the research procedures (Figure 1).

24 participants $(54.5 \%)$ had the middle cerebral artery syndrome and $59.5 \%$ had a right-brain stroke. The participants told that HIT was suitable without serious adverse effects. Estimating the standard effect size for the result of most measures between the groups was moderate to enormous

Figure 2 shows the mean and standard deviation (SD) of the NIHSS scores. Figure 3 presents the mean MRS scores and Figure 4 shows the MMSE scores for the two intervention groups (alternative and continuous aerobic exercise, and without exercise group), before and after the intervention program. According to the results of the ANOVA posttest and Tukey post hoc test, the NIHSS scores reduced in both exercise groups compared to the control one, in the post-intervention period. This reduction was marked with the $\mathrm{SD}$ of 1.000 in the alternative exercise group. Besides, the MMSE score increased for the participants in the continuous exercise group, while the MRS score reduced. These differences were statistically significant $(P<0.05)$.

Table 1 The participants' qualitative and descriptive demographic characteristics

\begin{tabular}{|c|c|c|c|c|}
\hline $\begin{array}{c}\text { Groups } \\
\text { Variable/index }\end{array}$ & $\begin{array}{c}\text { continuous } \\
\text { Training Grope }\end{array}$ & $\begin{array}{l}\text { high-intensity } \\
\text { anaerobic }\end{array}$ & Control Grope & p-value \\
\hline & Percent \% & Percent $\%$ & Percent \% & \multirow[b]{4}{*}{$>0 / 05$} \\
\hline \multicolumn{4}{|l|}{ gender } & \\
\hline Men & $12(80)$ & $11(73.3)$ & $11(73.3)$ & \\
\hline Women & $3(20)$ & $4(26.7)$ & $4(26.7)$ & \\
\hline Impaired limb injury & & & & \\
\hline Left side of the body & $5(35.7)$ & $6(0.40)$ & $7(46.7)$ & \multirow{3}{*}{$0 / 001^{*}$} \\
\hline Right side of the body & $10(64.3)$ & $9(60.0)$ & $8(53.6)$ & \\
\hline \multicolumn{4}{|l|}{$\underline{\text { Location of brain damage }}$} & \\
\hline Middle artery & $7(50)$ & $6(40)$ & $9(73.3)$ & \multirow{5}{*}{0.48} \\
\hline Posterior artery & $2(13.3)$ & $2(13.3)$ & $4(9.1)$ & \\
\hline Anterior artery & $1(7.1)$ & 0 & $3(20)$ & \\
\hline Lacunar section & $3(20.6)$ & $7(46.7)$ & $1(6.7)$ & \\
\hline \multicolumn{4}{|l|}{$\underline{\text { TIA Stroke. } H}$} & \\
\hline yes & $8(53.3)$ & $6(40)$ & $9(60)$ & \multirow[b]{2}{*}{$0.001^{8}$} \\
\hline No & $7(46.7)$ & $9(60)$ & $6(40)$ & \\
\hline
\end{tabular}

*P $<0.05$ 


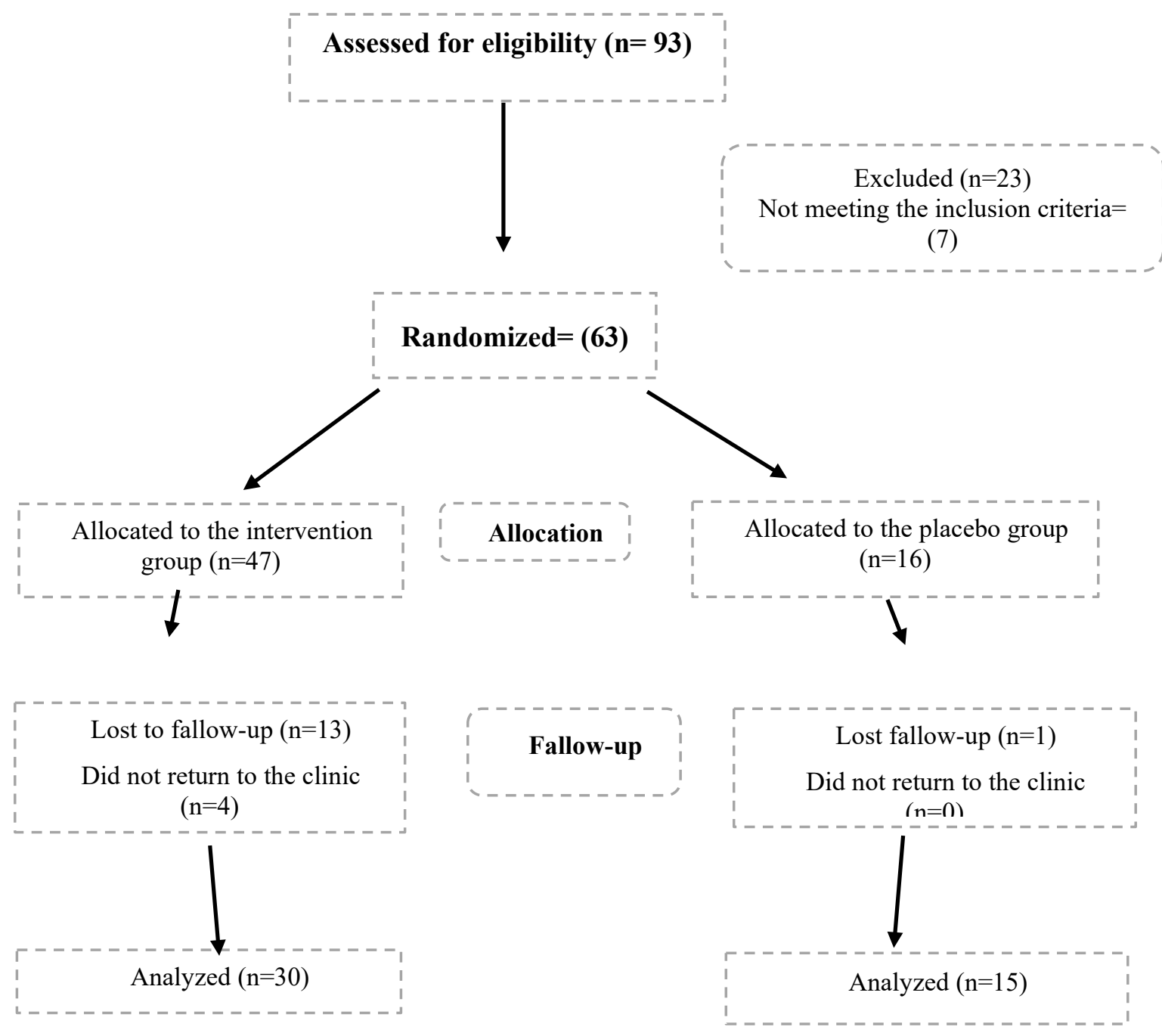

Figure 1. Study flow chart, a total 45 patients were studied (intervention groups 30 and control group 15)

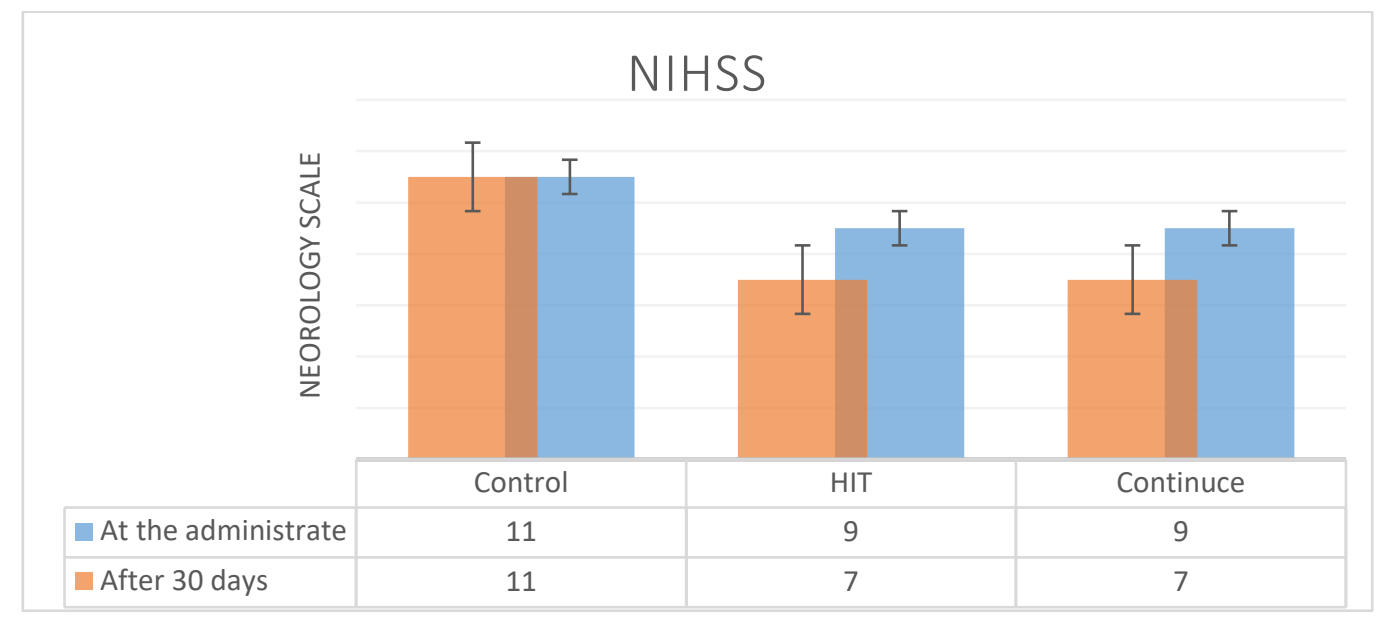

Figure2. The NIHSS mean and SD, before and after the training intervention show no changes in the control group at the administration (score 11); meanwhile in intervention group the score reduced (from 9 to 7 ) after 30 days. 


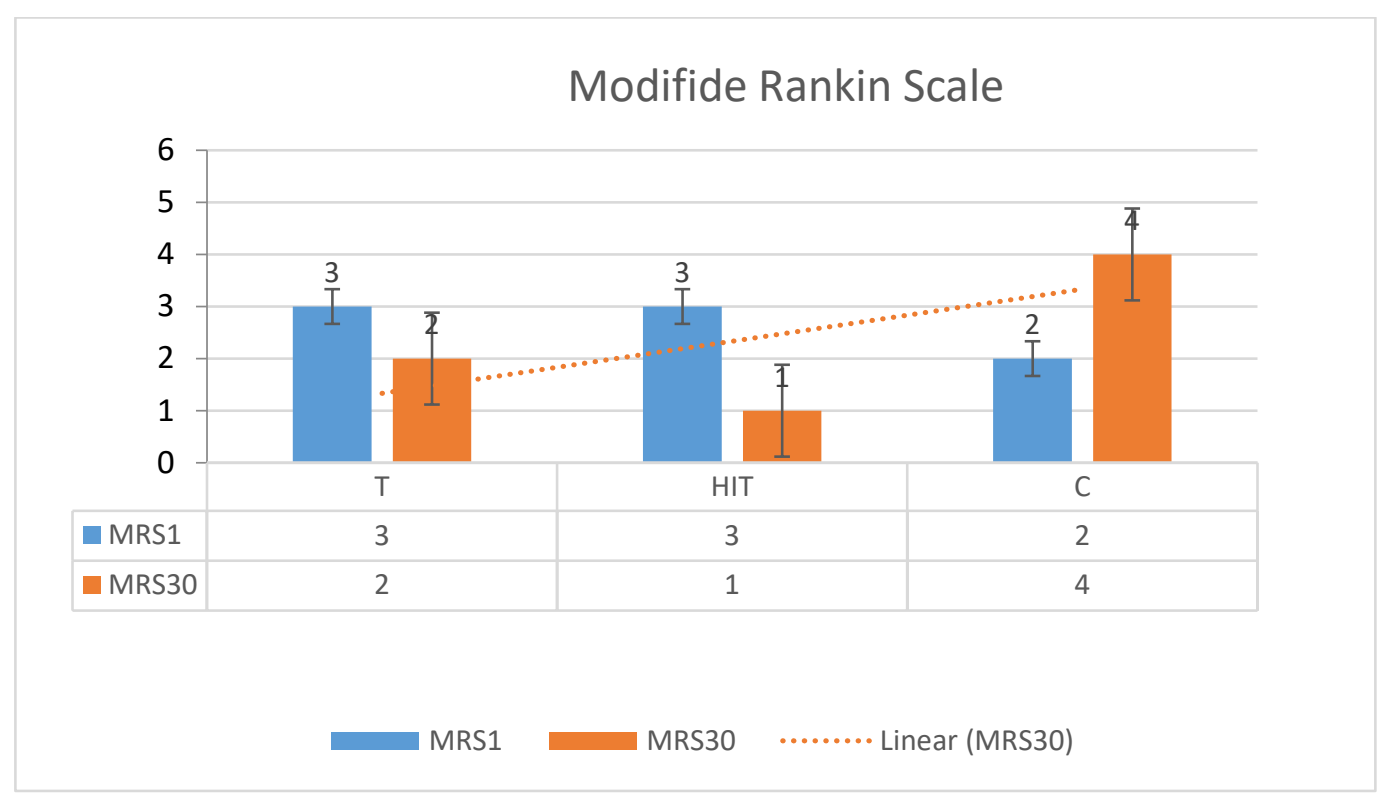

Figure3. The MRS mean and SD, before and after the training intervention (the chart shows a reduced MRS, from high number to the low number)

\section{Discussion}

The present study showed that exercise can help to reduce the effects of vascular damage by reduced NIHSS (from 9 to 7 ) in the intervention groups, after 30 days. Meanwhile, we did not find any changes in the control group (score 11). As the results showed, MRS reduced (from 4 to 2), MMSE increased (from 21.93 to 23.4) in HIT and in the continuous groups (from 22.4 to 24.14). Most people with CVA die in the first month after the stroke or become dependent on others $[1,2$, 22]. The stroke rehabilitation guidelines have recommended moderate continuous exercise training (MCT) to improve motor skills, aerobic capacity and cardiovascular health. However, only $6 \%$ of patients use the rehabilitation guidelines [15].

Aerobic exercise may dramatically alter the brain's response to rehabilitation and facilitate the stroke recovery conditions [10]. The effect of this aerobic preparation has been recognized through various mechanisms such as cutting pressure, contraction, muscle tension, cytokine secretion [7], VEGF changes [8] and brain-derived neurotrophic factor (BDNF) secretion [10], increased nitric oxide, reduction of blood lipids [1], $\mathrm{VO}_{2}$ max expansion and increased activity of aerobic enzymes [11]. However, the optimal exercise parameters to target brain mechanisms are not yet known. Nevertheless, evidence points out that higher-intensity exercises might be more useful than MCT for both aerobic and motor prognosis [1, 2].

HIT increases the intensity of exercise using maximum focused efforts alternating with recovery periods [18]. However, MCT is more effective to refine aerobic capability in both healthy adults and people with cardiac problems.
In the rehabilitation course of CVA patients in the hospital, HIT treadmills were more effective than additional treatments (based on neuromuscular facilitation and prophylactic specific neurodevelopment methods) [23]. They were more effective to improve the walking speed and spatial parameters than other forms of anaerobic exercise training [24].

A study on patients with chronic ischemic stroke showed that high-intensity treadmill training for more than six months resulted in significant improvements in the aerobic capacity of $\mathrm{VO}_{2}$ peak intake and metabolic walking rates. In addition to the walking speed, the walking time, sit-up scores and the sixminute walk scores were improved [25]. Therefore, in other activities, aerobic conditioning can be a major obstacle to improve cardiovascular events [1].

Boyne (2016) [26] compared the effects of HIT and MCT treating on people with chronic stroke. A total of 26 individuals in two groups were trained twenty-five minutes, three times a week for four weeks. The HIT training consisted of a thirty-second fitful exercise with a maximum tolerance treadmill speed and 30-60 second intermittent rest periods.

The MCT strategy included continuous treadmill walking at $45-50 \%$ of the heart rate. The increased metabolic walking rate led to a $30 \%$ improvement in the participants' tests results (ten-minute walking test and six-minute ground walking test). Continuous aerobic training (MCT) increases aerobic volume and agility after stroke. HIT is more effective than MCT on patients with chronic stroke. 
Following this study, two moderate and highintensity protocols were administered to 19 people with chronic ischemic stroke (Pierce et al., 2016) [13]. In this study, the two 40-60 minute continuous aerobic protocols were compared to the 30 -second intermittent aerobic protocol, with high intensity and repetition. Of the 44 participants, pain in the affected limbs was the main complaint of clients in both groups. The muscular strength of the lower limbs responded faster to the exercises than the upper limbs.

Besides, the data analysis using NIHSS, MMSE and MRS showed that the training intensity was sufficient to assess NIHSS scores in the intermittent training in the intervention groups compared to the control. It could facilitate the stimulation of the cerebral cortex. The results showed a significant reduction in the intermittent group with a SD of 1.000 at the significance level of $0.05(P<0.05)$. Besides, the MRS score decreased (from 3 to 2 ). It was also shown that the MMSE scores improved during the 28 days after the training. Continuous training was more effective than intermittent training for the participants.

The findings revealed the neural mechanisms of aerobic exercise, to choose the optimal dose of acute neurological effects. Similarly, Ghasemi (2010) [27] compared three different training methods performed on 30 patients with chronic ischemic stroke in the midcerebral artery. The patients experienced twelve sessions of forty five-minute with a frequency protocol of $40 \mathrm{~Hz}$ and $250 \mu$ s for two seconds. The rest time was set to be four seconds, until the contraction and elevation of the quadriceps muscle were observed. Then, the dorsiflexors were examined by the electrode, intermittent isometric contractions and overflow, rhythmic stabilizer and quadriceps setting exercise with the swiss ball. The results showed that biofeedback and exercise therapy had the greatest effects on the muscle strength and kinematic parameters of patients' gait.

A meta-analysis study was done on the muscles of patients over 18 years of age, with chronic stroke less than three months. Wist (2016) [28] has reported that advanced resistance training is the most effective factor in the traction of lower limb, walking distance and speed and strength increase and balance maintenance, compared to the stretching exercises, in the long time.

However, there was no clear result for walking, stamina. Sundseth (2012) [8] studied the role of early exercise training in 56 patients with acute stroke less than 24 hours after admission. They assessed the role of early initiation with the MRS scale, the degree of dependence on others with the Barthel scale and the mortality rate with the NHISS. The experimental group showed a better result after three months of early training than the control group.

It should be noted that the nervous system has not only mechanisms to control the muscle function, but also to affect the function of the brain structure.
Accordingly, physical activity can play an important role in the cognitive functions of the brain including learning and memory. A recent meta-analysis study by Lista (2013) [29] confirmed the major impact of exercise on cognitive functions. However, the underlying biological mechanisms for such useful effects are still unidentified.

Matsuda (2011) [12] divided 77 Wistar rats into three groups by inducing stroke in the middle artery (ischemic control: 36, ischemic-training: 36, and exercise group: 5). The mice exercised on the treadmill twenty minutes daily for maximum of 28 consecutive days. Improvements in mouse behaviors were observed after ischemia, using a beam walking test and a neural evaluation scale.

Holleran (2014) [30] conducted a pilot study on 25 participants with six-month chronic stroke, after four weeks of training with a walking speed of less than 0.9 $\mathrm{m} / \mathrm{s}$, and with minimal assistance as a step-by-step exercise in a variable context with $70-80 \%$ heart rate. The results showed that walking speed and six-minute walking were significantly improved after training and high-intensity aerobic exercise. Accordingly, exercise can help to reduce the effects of vascular damage by increasing blood flow to the muscles; it stimulates glutathione- 4 receptors in the muscle, as well as the secretion of adrenaline-stimulating hormones.

\section{Limitations}

One of the limitations of this study was the very low motivation of the patients to participate in the training sessions, and the fear of worsening the complications caused by cerebral infarction. The patients' dependence on the family was another limitation to participate in the training sessions.

\section{Suggestions}

Based on the findings of the study, training interventions should be performed over a period of more than four weeks. Besides, the effectiveness of the training program must be assessed by measuring related enzymes to muscle tissue. Moreover, clinical training centers should be considered for patients' rehabilitation, as soon as possible.

\section{Conclusion}

Performing intermittent exercise over four weeks can improve the overall quality of life of CVA patients by reducing the MRS score providing new insights into a better understanding of brain damages caused by cardiovascular ischemia. 


\section{Acknowledgments}

Acknowledgments: This research project was a clinical trial; it was extracted from the doctoral dissertation in the field of sports physiology. The authors appreciate the professors, patients and the staff of the laboratory and the emergency and neurology department of Loghman Hakim Hospital Medical Center in Tehran, for their assistance to conduct the study.

\section{Conflict of Interest}

The authors declared no conflict of interest.

\section{References}

1. Billinger SA, Arena $\mathrm{R}$, Bernhardt $\mathrm{J}$, et al. Physical activity and exercise recommendations for stroke survivors: a statement for healthcare professionals from the American Heart Association/American Stroke Association. Stroke. 2014;45:2532-53. [DOI:10.1161/STR.0000000000000022]

2. Hornby TG, Straube DS, Kinnaird CR, et al. Importance of specificity, amount, and intensity of locomotor training to improve ambulatory function in patients oststroke. Top Stroke Rehabil. 2011;18:293-307.

[DOI:10.1310/tsr1804-293]

3. Zhang Pe, Zhang QI, Hongjian Pu, et al. Very early - initiated physical rehabilitation protects against ischemic brain injury.Departman of rehabilitation of Huashan Hospital, Department of Neurology. University of Pittsburgh school of medicine.PA

15261,USA:

2015:https://www.researchgate.net/publication/2 69792216.doi:10.2741/E559. [PubMed] [Google Scholar]

4. Naghavi FS, Koffman E E, Lin B, Du Jy. Post Stroke neuronal circuits and mental illnesses. Int J Physiol Pathophysiol Pharmacol.PMC6420715.2019: 11(1): 1-11. [PubMed] [Google Scholar]

5. Jafari, M, Dalvandi, A. Quality of life of stroke survivors and its related factors. Iran J Nurs. 2014;87(27):

14-22. [DOI:10.29252/ijn.27.87.14]

6. Longo D, Fauci A, Kasper D, Hauser S. Harrison's Principles of Internal Medicine (2011).18th edition: McGraw-Hill Professional.

7. Lucas S, James D, Brassard P, Bailey D.. Highintensity interval exercise and cerebrovascular health: curiosity, cause, and consequence J Cereb Blood Flow \& Metab (2015): 35, 902-911. [DOI:10.1038/jcbfm.2015.49]
8. Sundseth A, Thommessen B, Rønning OM. Outcome after mobilization within 24 hours of acute stroke: A randomized controlled trial. Stroke. 2012;43(9):2389-94. [DOI:10.1161/STROKEAHA.111.646687]

9. Vanroy C, Feys P, Anke S, Yves V, et al.. Effectiveness of active cycling in subacute stroke rehabilitation: A randomized controlled trial Archives of Physical Medicine and Rehabilitation.2017;98(8):1576-85. [DOI:10.1016/j.apmr.2017.02.004]

10. Boyne P, Meyrose C, Westover J, Dustyn W. Exercise intensity affects acute neurotrophic and neurophysiological responses posts stroke. J Appl Physiol (1985). 2019;126(2):431-443. [DOI:10.1152/japplphysiol.00594.2018]

11. Tang K, Xia FC, Wagner PD, Breen EC.. Exercise-induced VEGF transcriptional activation in brain, lung and skeletal muscle. Respir Physiol Neurobiol. 2010; 170(1):16-22. [DOI:10.1016/i.resp.2009.10.007]

12. Matsuda F, Sakakima H, Yoshida Y. The effects of early exercise on brain damage and recovery after focal cerebral infarction in rats. Acta Physiol (Oxf). 2011 ; 201:275-87. [DOI:10.1111/j.1748-1716.2010.02174.x]

13. Pierce Boyne, Kari D, Daniel C, et al. Highintensity interval training and moderate-intensity continuous training in ambulatory chronic stroke: Published online 2016 [DOI:10.2522/ptj.20150277]

14. Askim T, Dahl AE, Aamot IL, et al. Highintensity aerobic interval training for patients 3-9 months after stroke: a feasibility study. Physiother Res Int. 2014;19:129-139. [DOI:10.1002/pri.1573]

15. Carolee J, Winstein, Joel Stein ,Ross Arena, Barbara Bates, Leora R. Cherney A guideline for healthcare professionals from the American Heart Association/American Stroke Association. Stroke. 2016;47:e 98-e169. [DOI:10.1161/STR.0000000000000098]

16. Pin-Barre C, Laurin, J.. Physiological adaptations following endurance exercises after stroke: Focus on the plausible role of high-intensity interval training . Int. J Phys Med Rehabil, 2014.S3006 [DOI:10.4172/2329-9096.S3-006]

17. Burgomaster KA, Howarth KR, Phillips SM,et al.. Similar metabolic adaptations during exercise after low volume sprint interval and traditional endurance training in humans. 2008; J Physiol; 586(1):151-60.

\section{[DOI:10.1113/jphysiol.2007.142109]}

18. Gibala Martini J, Little JP, Macdonald MJ, Hawley JA. Physiological adaptations to lowvolume, high-intensity interval training in health 
and disease. J Physiol. 2012;590:1077-1084. [DOI:10.1113/jphysiol.2011.224725]

19. Runge M, Hullmann K. (2011). Arm-ergometry in patients with cognitive impairments. B\&G Move Health Sport. 27(1):27-30. [PubMed] [Google Scholar]. [DOI:10.1055/s-00301262765]

20. Alastair Wi, Philip MW Bath, Eivind B. Understanding the relationship between costs and the modified modified Rankin Scale: A systematic review, multidisciplinary consensus and recommendations for future studies. Eur Stroke J . 2017;2(1):3-12. [PubMed] [Google Scholar].: [DOI:10.1177/2396987316684705.]

21. Adams H, Davis P, Hansen M, et al. (2017). Baseline NIH stroke scale score strongly predicts outcome after stroke : A report of the trial of org 10172 in acute stroke treatment (TOAST)".2017; Neurol. $\quad 53 \quad$ (1): 126-131. [DOI:10.1212/WNL.53.1.126]

22. Bonita R, Beaglehole R. Recovery of motor function after stroke. Stroke. 1988;19(12):1497500. [DOI:10.1161/01.STR.19.12.1497]

23. Pohl M, Mehrholz J, Ritschel C, Ruckriem S. Speed-dependent treadmill training in ambulatory hemiparetic stroke patients: a randomized controlled trial. Stroke. 2002;33:553-558.

[DOI:10.1161/hs0202.102365]

24. Lau KW, Mak MK. Speed-dependent treadmill training is effective to improve gait and balance performance in patients with sub-acute stroke. J Rehabil Med. 2011;43:709-713. [DOI:10.2340/16501977-0838]

25. Gjellesvik TI, Brurok B, Hoff J, et al. Effect of high aerobic intensity interval treadmill walking in people with chronic stroke: a pilot study with one year follow-up. Top Stroke Rehabil. 2012;19:353-360. [DOI:10.1310/tsr1904-353]
26. Boyne P, Dunning K, Carl D, et al. High-intensity interval training and moderate-intensity continuous training in ambulatory chronic stroke: Feasibil Study.2016;96(10):1533-44. [DOI:10.2522/ptj.20150277]

27. Ghasemi Shaygannejad V, Joker S, Rezaiean F, Armak M., Mahmoodi Z .(2010). The effectiveness of functional electrical stimulation (FES), biofeedback and exercise therapy on various parameters of lower limb muscle strength , kinematics of gait and balance in stroke patients: A comparative study .J Res Rehabil Sci.2010; 6(1).

28. Wist S, Julie Clivaz,J, Sattelmayer M. Muscle strengthening for hemiparesis after stroke: A meta-analysis. Ann Physic Rehabil Med.2016; 59: 114-24. [DOI:10.1016/j.rehab.2016.02.001]

29. Lista I, Sorrentino G. (2013). Biological mechanisms of physical activity in preventing cognitive decline. Cell Mol Neurobiol.2013; 30(4):493-503. [DOI:10.1007/s10571-009-9488$\underline{x}]$

30. Holleran Carey L, Don D, Straube PT,et al. (2014). Feasibility and potential efficacy of highintensity stepping training in variable contexts in subacute and chronic stroke. Neurorehabil Neural Repair. 2014; 28(7): 643-51. [DOI:10.1177/1545968314521001]

31. Swain D, Lutholtz B. Metabolic calculations (Applied Equations). Translated by Raeesi Abdoreza, Dana Publishing.pp.108-110.2001[ Persion Text Booke].

\section{How to Cite This Article:}

Kadkhodaei Khalafi M, Simani L, Shojaei M, Hajiesmaeili M. Comparison of the Effect of Exercise Type on the Prognosis Scores in Patients with Acute Ischemic Stroke. J Adv Med Biomed Res. 2021; 29 (135) :215-222

\section{Download citation:}

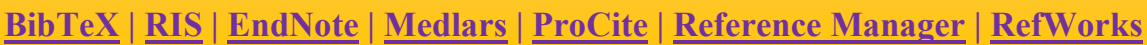

\section{Send citation to:}
(3). Mendeley
$\underline{\text { Zotero }}$ RefWorks RefWorks 